\title{
A Spectroscopic Survey of Recurrent Novae at Minimum ${ }^{1}$
}

\author{
H.W. Duerbeck and W.C. Seitter \\ Astronomisches Institut der Westfälischen Wilhelms-Universität \\ Münster, F.R. Germany
}

From 1986 to 1988 optical spectroscopy near minimum of all then established recurrent novae (excluding recurrent X-ray novae such as V616 Mon and V404 Cyg) was carried out with the $3.6 \mathrm{~m}$ and $1.5 \mathrm{~m}$ telescopes of ESO, using EFOSC with CCD and the Cassegrain spectrograph with CCD or IDS, respectively. Flux-calibrated spectra of the six objects are shown in Figs. $1 \mathrm{a}-\mathrm{f}$. The most recent recurrent nova, V745 Sco, is included in the present discussion, because 1 month after outburst it had already reached a very late stage of outburst evolution. A spectrum of V745 Sco is shown in Schwarz et al. (1989).

The small number of RNe does not allow a proper statistical investigation, however, one can try to group the objects according to some physical criteria. The sequence given in Table 1 (column 1) runs from late-type dominated to early-type dominated spectra. This criterion depends both on the spectral type of the late-type star (column 3 ) and the relative strength of the blue source. In several systems, contributions from both continua are found, which implies that the red and the blue sources are of comparable strength. When the ratio $\mathrm{He} \mathrm{II} / \mathrm{H} \beta$ (column 4 ), which measures the temperature of the hot source, is the chosen criterion, the sequence starts with the last three objects in Table 1 and ends with the late-type dominated stars. Using the modified Zanstra relation given by Kenyon (1986), the stellar temperatures of the primary components of T Pyx, U Sco and V394 CrA are of the order $10^{5} \mathrm{~K}$. V1017 Sgr is cooler than $50000 \mathrm{~K}$. The other stars appear to be much cooler unless the hydrogen emission originates in the envelope of the cool component, in which case the temperature of the primary component cannot be determined from the line ratio. The structure of the H-lines suggests this for objects with late M-type companions.

Density criteria reveal a further relation. Using as indicator the relative strength of He I $587.6 \mathrm{~nm}$ to He I $667.8 \mathrm{~nm}$ (Schmid 1989), where the triplet line is stronger at electron densities above $10^{7} \mathrm{~cm}^{-3}$, the combinations cool/tenuous and hot/dense envelopes might be be prevalent.

It should be noted that V394 CrA and U Sco, whose minimum properties agree very well, also had very similar outbursts (Sekiguchi et al. 1988, 1989).

A final remark concerns the time scales of outbursts. The average time between outbursts of all $\mathrm{RNe}$ is $30 \mathrm{yr} \pm 19 \mathrm{yr}$. Since more than half of all novae were discovered during the past 50 years we may be in for some surprises, such as the ones of V394 CrA and V745 Sco.

TABLE 1. Properties of Recurrent Novae

\begin{tabular}{|l|l|c|c|c|}
\hline \multicolumn{1}{|c|}{ object } & \multicolumn{1}{|c|}{ years of outburst } & $\begin{array}{c}\text { spectral type } \\
\text { of secondary }\end{array}$ & $\frac{\text { He II 468.6 }}{\text { H } \beta 486.1}$ & $\frac{\text { He I 587.6 }}{\text { HeI 667.8 }}$ \\
\hline V745 Sco & 1937,1989 & M 6-6.5 III & $?$ & $?$ \\
T CrB & 1866,1946 & M 5 III & $\ll 1$ & $?$ \\
RS Oph & $1898,1933,1958,1967,1985$ & K 5-7 III & $\ll 1$ & $\gg 1$ \\
V1017 Sgr & $1901,1919,1973$ & G 9 III & $<1$ & $>1$ \\
V394 CrA & 1949,1987 & K & $\gg 1$ & $?$ \\
U Sco & $1863,1906,1936,1979,1987$ & $?$ & $\gg 1$ & $<1 ?$ \\
T Pyx & $1890,1902,1920,1944,1966$ & $?$ & $>1$ & $\approx 1$ \\
\hline
\end{tabular}

\footnotetext{
${ }^{1}$ Based on observations collected at the European Southern Observatory, La Silla, Chile
} 


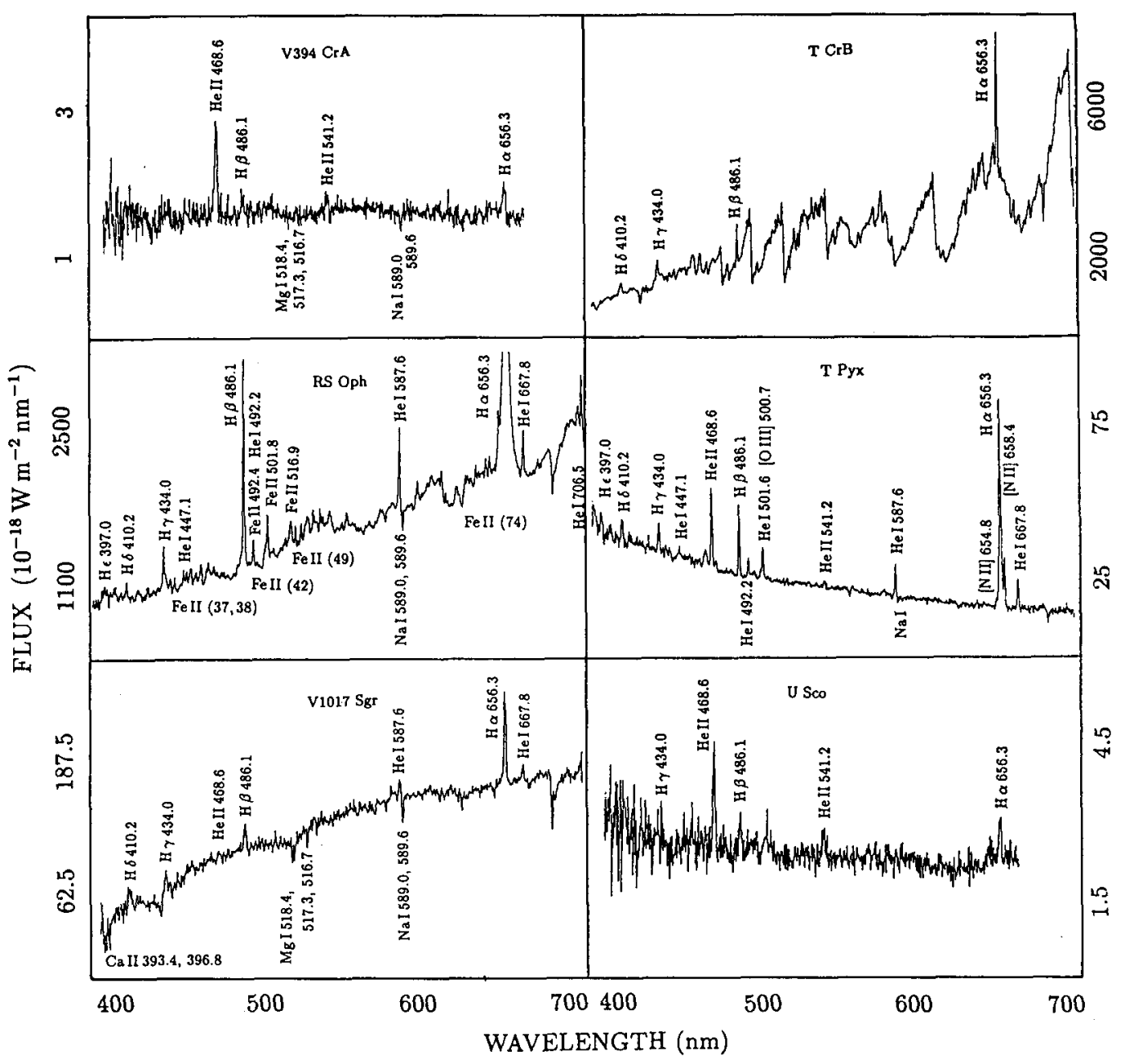

Fig. 1. Spectra of recurrent novae at minimum.

\section{Acknowledgement}

Thanks go to R. Duemmler and H.-J. Tucholke for critically reading the manuscript and to M. Tacke for editorial help.

\section{References}

Kenyon, S.J., 1986. The Symbiotic Stars. Cambridge: Cambridge University Press.

Schmid, H.M., 1989. These proceedings.

Schwarz, H.E., Duerbeck, H.W., Seitter, W.C., 1989. These proceedings.

Sekiguchi, K., Catchpole, R.M., Fairall, A.P., Feast, M.W., Kilkenny, D., Laney, C.D., Lloyd Evans, T., Marang, F., Parker, Q.A., 1989. Mon. Not. R. astr. Soc. (in press).

Sekiguchi, K., Feast, M.W., Whitelock, P.A., Overbeek, M.D., Wargau, W., 1988. Mon. Not. R. astr. Soc., 234, 281. 